\title{
Diffuse large B-cell lymphoma in and of the bone. Forms and potential precursors
}

\section{Abstract}

Primary diffuse large B-cell lymphoma of the bone (PB-DLBCL) is a rare event and is a diagnosis by exclusion of secondary bone involvement. Here we review the literature regarding PB-DLBCL and give essentials for this diagnosis. Furthermore, we give an overview of potential precursors and discuss chronic recurrent multifocal osteomyelitis (CRMO) as a potential risk factor for $\mathrm{PB}-\mathrm{DLBCL}$ development in the young.

\section{Primary Bone Diffuse Large B-cell Lymphoma (PB-DLBCL)}

Primary bone non-Hodgkin lymphoma, other than plasmocytic myeloma and solitary plasmacytoma of bone, is defined by the World Health Organization (WHO) as a neoplasm composed of malignant lymphoid cells, with one or more bone lesions; by definition, lymph node or other extranodal lesions are not involved [1]. Primary lymphoma of bone is rare and accounts for less than $2 \%$ of adult lymphomas [2] and approximatively for $7 \%$ of malignant bone tumors. Between 70 and $80 \%$ of primary bone lymphomas are classified as diffuse large B-cell lymphoma (DLBCL), the rest are anaplastic large, mostly ALK-positive T-cell lymphomas [3-5]. These aggressive lymphomas have preference for the axial skeleton and long tubular bones. The most commonly affected bones are femur, ilium, vertebrae, and humerus. PB-DLBCL is exceedingly rare in the young and tends to occur at the age of 45 while the peak incidence is in the range of 75 to 79 years [6].

Main symptoms are pain, local tenderness, redness, swelling, and nerve compression (related to the tumor location). Pathological fractures occur in $10-20 \%$ of PB-DLBCL, and, therefore, this is the most frequent complication at presentation of primary bone lymphoma [7].
The standard treatment for all kinds of DLBCL is the R-CHOP (Rituximab immunotherapy, i.e., an anti CD20 monoclonal antibody), combined with cyclophosphamide, doxorubicin hydrochloride, vincristine, and prednisone), the total number of cycles and addition of radiation therapy depending on tumor bulk [8].

DLBCL is a neoplasm of diffusely arranged medium or large lymphoid B-cells with nuclei more than twice the size of normal lymphocytes nuclei featuring open chromatin [9]. These lymphomas are CD20 + ( Figure 1 A-D), CD3-, CyclinD1-, CD138and have a high proliferation (KI-67) index generally above $50 \%$. Traditionally, DLBCLs were further characterized by cytomorphology as centroblastic, immunoblastic, plasmoblastic, and anaplastic. The current WHO classification still lists these variants, however, has promoted the transciptomebased molecular subgrouping with the two major subtypes activated B-cell-like (ABC) and germinal-center B-cell-like (GCB); by immunohistochemistry, this subdivision can be approximately done using an algorithm proposed by Hans following the expression of CD10, Bcl-6, and MUM-1 [10]. More recently, multiplatform genetic analysis allowed the identification of five subtypes of DLBCL making a further step in the direction of precision medicine [11, 12]. PB-DLBCL has been found to be predominantly of the $A B C$ type [13]. Regarding bone as primary DLBCL location, the clinical significance of multifocal bone involvement has been investigated, in comparison with unifocal primary and secondary bone lymphoma. Multifocal primary bone DLBCL is very rare and constitutes around $3-10 \%$ of cases in the largest reported series of primary bone lymphomas [14]. Findings of Wu et al. (2014) suggest a major similarity between multifocal primary bone and secondary involvement rather than with unifocal DLBCL of the bone [15]. On the other hand, in the study of Messina et al. (2014) prognosis of multifocal primary bone DLBCL is significantly better than bone involvement at advanced stages of systemic DLBCL [14].

The relationship between chronic inflammation and lymphoma development is well established for marginal B-cell lymphoma of the salivary gland and Sjögrens' disease, of lacrimal gland and chlamydial dacryoadenitis, of gastric marginal B-cell lymphoma and chronic gastritis, e.g., due to $H$. pylori infection, and for gluten-sensitive enteropathy-associated intestinal T-cell lymphoma [9]. The relationship is far less clear, if at all existing for chronic inflammation of the bone and subsequent lymphoma development.

We here list anecdotal observations suggesting such a relationship.

- Rheumatoid arthritis: Patients with rheumatoid arthritis (RA) are at a double risk of developing malignant lymphoma and studies and case series of RA-associated lymphomas indicate an increased proportion of diffuse large Bcell lymphomas (DLBCLs) in RA patients [16].

- Implantation of cobalt-chromiummolybdenum alloy joint prosthesis: The coincidence of PB-DLBCL was described in a patient during a surgical revision of the loosened prosthesis. Debris in the periprosthetic membrane are antigenic and lead to chronic inflammation through macrophage activation [17]. The pathogenesis has also been correlated with the release of chromium and cobalt ions from the prothesis [18].

- Chronic recurrent multifocal osteomyelitis (CRMO): There are different case reports on patients diagnosed with bone DLBCL after years of CRMO, especially in younger patients.

- Moreover, infectious osteomyelitis in general has been seen associated with 
other types of tumor, e.g., carcinoma and sarcoma and even DLBCL $[19,20]$ Asif et al. in 2019 describe the case of a 75 years old patients, diagnosed with osteomyelitis caused by Raoultella planticola infection and subsequent discovery of DLBC [21]. Senthilvel et al. in 2011 report on a 51 years old patient, with a rare primary $D L B C L$ of the skull that was distinguished from osteomyelitis only by biopsy of the tumor mass. The authors state that this peculiar site made the formulation of the diagnosis difficult [22]. Romero-Rojas in 2014 described in a 27 years old patient, a DLBCL after three years of diagnosis of chronic osteomyelitis, following a long asymptomatic period. The authors conclusions point to the rare association between chronic inflammation and DLBCL, associated with EBV infection, metallic bone implants or Paget's disease [20].

\section{Chronic Recurrent Multifocal Osteomyelitis}

Osteomyelitis in its typical form is an inflammatory state caused by infection, the most common agent being $S$. aureus followed by Propionibacterium sp. [23], Pseudomonas aeruginosa [24], C. albicans, and Aspergillus sp. [25].

By contrast, chronic recurrent multifocal osteomyelitis (CRMO) is a rare, but most severe variant in the spectrum of chronic non-bacterial osteomyelitis, generally regarded as autoinflammatory disorders [26]. CRMO typically occurs in children and young adults. The most commonly affected sites are the diaphysis of the tibia and the femurs as well as the clavicle; CRMO may present symmetrically, since involvement of similar sites in both extremities have been described [27]. Main symptoms are fever, local swelling, and pain in the affected bones, sometimes accompanied by redness and skin inflammation [6].

Treatment for CRMO usually involves nonsteroidal anti-inflammatory drugs, corticosteroids, anti-rheumatic drugs, anti-TNF agents, or bisphosphonates. The choice of treatment relied for many years on the expert experience instead of empiric case data collection [28]. Only recently the Childhood Arthritis and Rheumatology
Research Alliance [29] defined treatment guidelines for chronic non-bacterial osteomyelitis and CRMO. The first line treatment consists in non-steroidal anti-inflammatory drugs (NSAIDs), while for second line therapy different options are defined: non-biological disease modifying anti-rheumatic drugs (DMARDs), TNF- $\alpha$ inhibitors with or without methotrexate or bisphosphonate [30].

The main pathophysiological mechanism of CRMO is thought to be a deregulation of pro- and anti-inflammatory cytokine balance, and the major players being IL-10 and overactivation of the inflammasome [31]

\section{Difficulties in diagnosis of CRMO and bone DLBCL}

For diagnosis of bone disorders acquisition of sufficient material is a prerequisite; however, needle bone biopsy may provide little amounts of material and is especially challenging in the long bones, while surgical biopsy is an invasive procedure. For this reason the main initial diagnostic tools are imaging technique, starting from standard radiography to higher resolution technique as PET [32] and MRI [33].

Regarding symptoms, CRMO and PB-DLBCL are largely overlapping with pain, swelling, and fracture. However, histology and immunohistology sufficiently define lymphoma. Absence of sheets of blastic cells, a high content of small lymphocytes and plasma cells in the absence of granulocytes characterize chronic osteomyelitis ( $\triangleright$ Figure $2 \mathrm{~A}-\mathrm{F}$ ). In doubtful situations, an immunoglobulin heavy chain rearrangement analysis may be done and is decisive since DLBCL is clonal and CRMO is not.

\section{Is there any relation between primary bone DLBCL and CMRO?}

Reviewing the literature, we found a few anecdotal observations of primary bone DLBCL discovered even years after diagnosis of CRMO.

Sulko et al. (2013) report the case of a 15 years old patients, first diagnosed as a CRMO, however, 20 months after the first symptoms the diagnosis of DLBCL was put forward on the basis of a biopsy of a supraclavicular lymph node, while a previous bone biopsy did not show evidences of DLBCL. In the article the authors show the possibility of misdiagnosis of two independent pathologic work-ups and speculate on a causative correlation between CRMO and DLBCL [19].

Jellicoe et al. (2008) describe a B-cell lymphoma in a 15 years old boy that arose adjacent to the site of biopsy of documented CRMO with a delay of 3.5 years. Original biopsies were reexamined after the lymphoma diagnosis but the review confirmed the absence of malignancy in the initial biopsy [34].

In the cases cited, however, it is interesting to note that initially the therapy for CRMO had a positive effect on the patient and that DLBCL seemed to emerge in a second step.

This is consistent with the hypothesis regarding a possible correlation between osteomyelitis and malignancies, such as $\mathrm{DLBCL}$, in a chronic inflammation context. With all these findings it may be tempting to speculate that the chronic inflammation
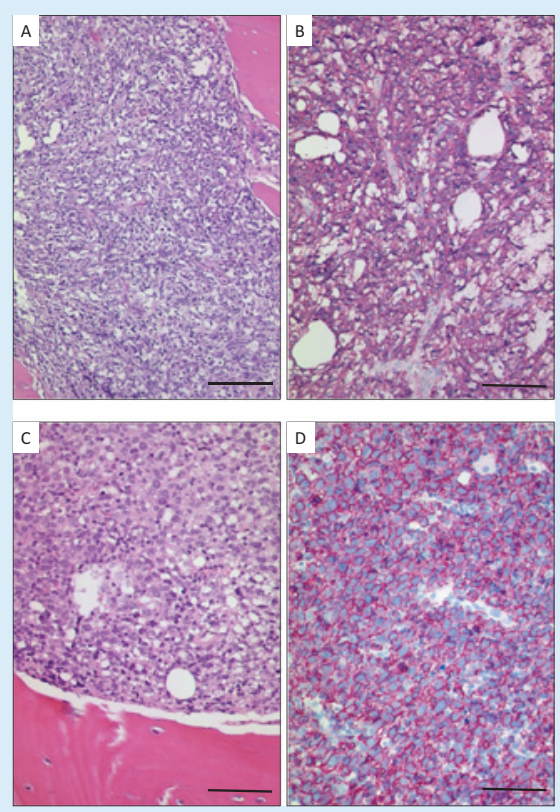

- Figure 1 Examples of Primary Bone Diffuse Large B-cell Lymphoma (Hematoxylin/Eosin staining; A, C). By immunohistochemical staining the lymphomas are strongly positive for CD20 (B, D). Scale $\operatorname{bar}(A)=200 \mu \mathrm{m}$. Scale bar $(B, C, D)=$ $100 \mu \mathrm{m}$. 

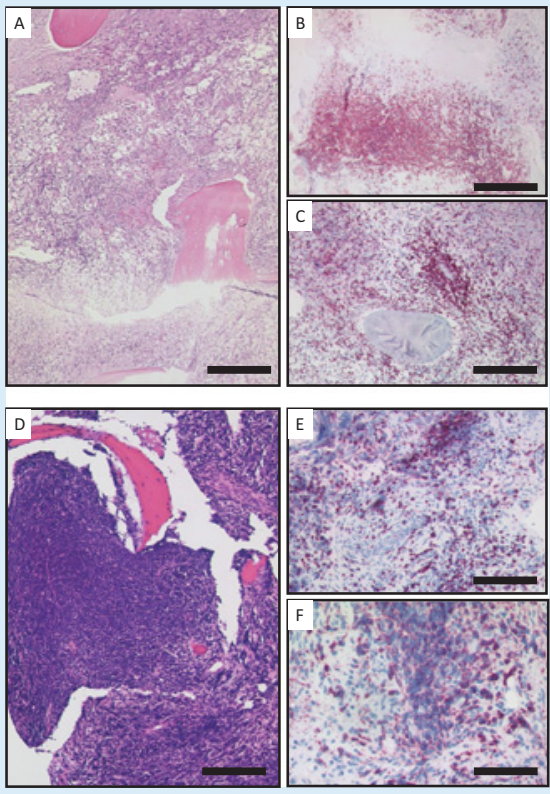

-Figure 2 Histology of Chronic Recurrent Multifocal Osteomyelitis of 15 years old boy in the acetabulum (A-C) and heel (D-F); Hematoxylin/Eosin staining (A, D) and immunohistochemistry staining of CD20 for B-cells (B, E) and CD3 for T-cells (C, F). Scale bar $(A)=500 \mu \mathrm{m}$. Scale bar $(B, C, D, E, F)=200 \mu \mathrm{m}$.

caused by CRMO paves the way for the emergence of primary bone DLBCL. Arguing long this line a tight and long lasting monitoring of CRMO patients seems advisable [6].

In conclusion, diagnosis of primary DLBCL of the bone is a histological diagnosis always needing the confirmation by extensive clinical staging that excludes any kind of systemic disease. For histology, a panel of antibodies including CD20, CD3, BCL-6, CD10, CD138, and MUM1 are essential. CRMO is a differential diagnosis which is excluded by histology, immunohistology and by immunoglobulin rearrangement showing a polyclonal pattern [35]. In case of diagnostic difficulties, a reference center for hematopathology should be consulted.

\section{Authors}

Francesca Spada, Thomas Breining, Thomas F. E. Barth and Peter Möller
Correspondence

\section{Peter Möller}

Institute of Pathology

University of Ulm

Albert-Einstein-Allee 11

$89081 \mathrm{Ulm}$

Germany

E-Mail: Peter.moeller@uniklinik-ulm.de

\section{References}

[1] WHO Classification of Tumours Editorial Board: Soft Tissue and Bone Tumours, Lyon (France) International Agency for Research on Cancer; 2020 (WHO Classification of Tumours series, 5th ed; vol. 3) https://publications.iarc.fr/588

[2] Bindal P, Desai A, Delasos L et al. Primary Bone Lymphoma: A Case Series and Review of Literature. Case Rep Hematol 2020; 2020: 4254803. doi: $10.1155 / 2020 / 4254803$

[3] Alencar A, Pitcher D, Byrne G et al. Primary bone lymphoma the university of miami experience. Leuk Lymphoma 2010; 51 (1): 3949. doi:10.3109/10428190903308007

[4] Jawad MU, Schneiderbauer MM, Min ES et al. Primary Lymphoma of Bone in Adult Patients. doi:10.1002/cncr.24828

[5] Ramadan KM, Shenkier T, Sehn LH et al. A clinicopathological retrospective study of 131 patients with primary bone lymphoma: A population-based study of successively treated cohorts from the British Columbia Cancer Agency. Ann Oncol 2007; 18: 129-135. doi:10.1093/annonc/mdl329

[6] Czerniak B. Dorfman and Czerniak's Bone Tumors2nd. Elsevier; 2015. Accessed November 2, 2020. https://www.elsevier.com/books/do rfman-and-czerniaks-bone-tumors/czerniak/9 78-0-323-02396-2.

[7] Govi S, Christie D, Messina C et al. The clinical features, management and prognostic effects of pathological fractures in a multicenter series of 373 patients with diffuse large B-cell lymphoma of the bone. Ann Oncol 2014; 25 (1): 176-181. doi:10.1093/annonc/mdt482

[8] Liu Y, Barta SK. Diffuse large B-cell lymphoma: 2019 update on diagnosis, risk stratification, and treatment. Am J Hematol 2019; 94 (5): 604-616. doi: 10.1002/ajh.25460

[9] WHO classification of tumours of haematopoietic and lymphoid tissues. ci.nii.ac.jp. Accessed November 2, 2020. https://ci.nii.ac. jp/naid/20001195667/

[10] Alizadeh AA, Elsen MB, Davis RE et al. Distinct types of diffuse large B-cell lymphoma identified by gene expression profiling. Nature 2000; 403 (6769): 503-511. doi:10.1038/ 35000501

[11] Chapuy B, Stewart C, Dunford AJ et al. Molecular subtypes of diffuse large B cell lymphoma are associated with distinct pathogenic mechanisms and outcomes. Nat Med 2018; 24 (5): 679-690. doi:10.1038/ s41591-018-0016-8

[12] Schmitz R, Wright GW, Huang DW et al. Genetics and Pathogenesis of Diffuse Large BCell Lymphoma. N Engl J Med 2018; 378 (15): 1396-1407. doi:10.1056/NEJMoa1801445

[13] Adams H, Tzankov A, Jundt $G$ et al. Primary diffuse large B-cell lymphomas of the bone: Prognostic relevance of protein expression and clinical factors B. Hum Pathol 2008; 39(9): 1323-30. doi: 10.1016/j. humpath.2008.01.004

[14] Messina C, Ferreri AJM, Govi S et al. Clinical features, management and prognosis of multifocal primary bone lymphoma: A retrospective study of the international extranodal lymphoma study group (the IELSG 14 study). $\mathrm{Br}$ J Haematol 2014; 164 (6): 834-840. doi:10.1111/bjh. 12714

[15] Wu H, Bui MM, Leston DG et al. Clinical Characteristics and Prognostic Factors of Bone Lymphomas: Focus on the Clinical Significance of Multifocal Bone Involvement by Primary Bone Large B-Cell Lymphomas BMC Cancer 2014; 14: 900. doi: 10.1186/14712407-14-900

[16] Baecklund E, Backlin C, lliadou A et al. Characteristics of diffuse large B cell lymphomas in rheumatoid arthritis. Arthritis Rheum 2006; 54 (12): 3774-3781. doi:10.1002/art.22277

[17] Cheuk W, Chan ACL, Chan JKC et al. Metallic Implant-Associated Lymphoma. Am J Surg Pathol 2005; 29 (6): 832-836. doi:10.1097/01. pas.0000157747.10967.f4

[18] Lidgren L. Chronic inflammation, joint replacement and malignant lymphoma. J Bone Jt Surg - Ser B 2008; 90 (1): 7-10. doi:10.1302/ 0301-620X.90B1.19823

[19] Sulko J. Diagnostic difficulties: Chronic recurrent multifocal osteomyelitis and lymphoma. One or two diseases? J Pediatr Orthop Part B 2013; 22 (2): 170-174. doi:10.1097| BPB.0b013e328354b481

[20] Romero-Rojas AE, Diaz-Perez JA, Raju S et al. Primary diffuse large B-cell lymphoma associated with chronic osteomyelitis of the knee. Knee 2014; 21 (6): 1280-1283. doi:10.1016/ j.knee.2014.08.016

[21] Asif S, Abughanimeh OK, Husainat NM et al. Maxillary Osteomyelitis with an Incidental Diagnosis of Maxillary Diffuse Large B-Cell Lymphoma: A Case Report. Cureus 2019; 11 (7). doi:10.7759/cureus.5238

[22] Senthilvel E, Subbiah S, Jain V et al. Primary diffuse large B-cell lymphoma of the skull mimicking osteomyelitis. Ear Nose Throat J 2011; 90 (1). doi:10.1177/014556131109000117

[23] Lew DP. Osteomyelitis. thelancet.com 2004; 364: 369. doi:10.1016/50140-6736(04) 16727-5

[24] Rao N, Ziran BH, Lipsky BA. Treating osteomyelitis: Antibiotics and surgery. Plast Reconstr 
Surg 2011; 127 (SUPPL. 1 S). doi:10.1097/ PRS.0b013e3182001fof

[25] Bariteau JT, Waryasz GR, McDonnell M et al. Fungal Osteomyelitis and Septic Arthritis. J Am Acad Orthop Surg 2014; 22 (6): 390401. doi:10.5435/JAAOS-22-06-390

[26] Roderick MR, Shah R, Rogers V et al. Chronic recurrent multifocal osteomyelitis (CRMO) advancing the diagnosis. Pediatr Rheumatol 2016; 14: 1. doi:10.1186/s12969-016-0109-1

[27] Meyer zu Reckendorf G, Milon E, Pous JG. Chronic recurrent multifocal osteomyelitis (C. R.M.O.). A case report and review of literature. Eur J Pediatr Surg 1996; 6 (5): 312-5. doi: 10.1055/s-2008-1071006

[28] Hofmann SR, Kapplusch F, Girschick HJ et al. Chronic Recurrent Multifocal Osteomyelitis (CRMO): Presentation, Pathogenesis, and
Treatment. Curr Osteoporos Rep 2017; 15 (6): 542-554. doi:10.1007/s11914-017-0405-9

[29] CARRA - Childhood Arthritis and Rheumatology Research Alliance. Accessed November 12, 2020. https://carragroup.org/

[30] Zhao Y, Wu EY, Oliver MS et al. Consensus Treatment Plans for Chronic Nonbacterial Osteomyelitis Refractory to Nonsteroidal Anti-Inflammatory Drugs and/or with Active Spinal Lesions HHS Public Access. Arthritis Care Res 2018; 70 (8): 1228-1237. doi:10.1002/ acr.23462

[31] Hofmann SR, Kubasch AS, loannidis C et al. Altered expression of IL-10 family cytokines in monocytes from CRMO patients result in enhanced IL-1 $1 \beta$ expression and release. Clin Immunol 2015; 161 (2): 300-307. doi:10.1016/ j.clim.2015.09.013
[32] Behzadi AH, Raza SI, Carrino JA et al. Applications of PET/CT and PET/MR Imaging in Primary Bone Malignancies. PET Clin 2018; 13 (4): 623-634. doi:10.1016/j.cpet.2018.05.012

[33] Kitsoulis P, Vlychou M, Papoudou-Bai A et al. Primary Lymphomas of Bone. Anticancer Res 2006; 26: 325-338

[34] Jellicoe P, Hopyan S. Can chronic recurrent multifocal osteomyelitis predispose to lymphoma of bone? A case report. J Pediatr Orthop B 2008; 17 (6): 329-332. doi:10.1097/ BPB.0b013e32830cc390

[35] Hummel M, Oeschger S, Barth FE et al. Wotherspoon criteria combined with B cell clonality analysis by advanced polymerase chain reaction technology discriminates covert gastric marginal zone lymphoma from chronic gastritis. Gut 2006; 55: 782-787. doi:10.1136/gut.2005.080523 